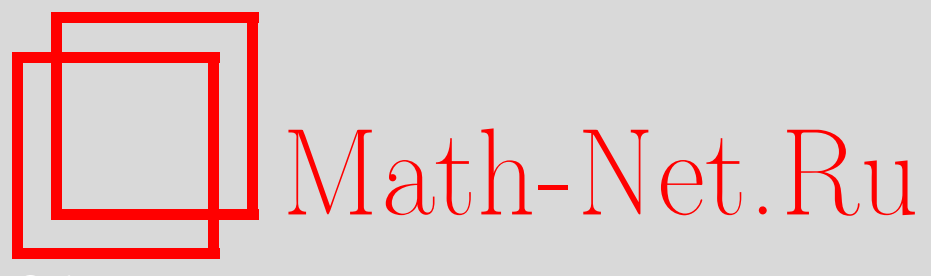

М. В. Шамолин, Случай полной интегрируемости в динамике на касательном расслоении двумерной сферы, УМH, 2007, том 62, выпуск 5, 169-170

DOI: https://doi.org/10.4213/rm7487

Использование Общероссийского математического портала Math-Net.Ru подразумевает, что вы прочитали и согласны с пользовательским соглашением http://www . mathnet.ru/rus/agreement

Параметры загрузки:

IP : 54.237 .206 .68

26 апреля 2023 г., $16: 24: 38$

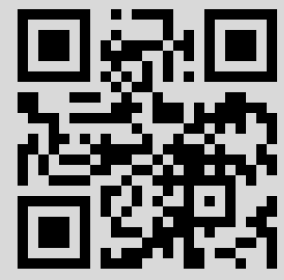




\section{Случай полной интегрируемости в динамике на касательном расслоении двумерной сферы}

\section{М. В. Шамолин}

В работе предлагается новый подход, позволяющий получать интегрируемые случаи в динамике свободного твердого тела, а именно интегрировать динамические уравнения, вложенные в $\mathbb{R}^{3} \times s o(3)$, в неконсервативном (диссипативном) поле сил.

1. Редуцированные динамические уравнения. Известные редуцированные динамические уравнения движения динамически симметричного твердого тела в диссипативном поле сил [1] имеют следующий вид:

$$
\begin{gathered}
\alpha^{\bullet} \nu \cos \alpha \cos \beta_{1}-\beta_{1}^{\bullet} \nu \sin \alpha \sin \beta_{1}+\Omega_{z} \nu \cos \alpha-\sigma \Omega_{z}^{\bullet}=0, \\
\alpha^{\bullet} \nu \cos \alpha \sin \beta_{1}+\beta_{1}^{\bullet} \nu \sin \alpha \cos \beta_{1}-\Omega_{y} \nu \cos \alpha+\sigma \Omega_{y}^{\bullet}=0, \\
I_{2} \Omega_{y}^{\bullet}=-z_{N} s(\alpha) \nu^{2}, \quad I_{2} \Omega_{z}^{\bullet}=y_{N} s(\alpha) \nu^{2} .
\end{gathered}
$$

Здесь $\operatorname{diag}\left\{I_{1}, I_{2}, I_{2}\right\}$ - тензор инерции, $\left(\nu, \alpha, \beta_{1}\right)$ - сферические координаты характерной его точки, $|\vec{\nu}|=\nu \equiv$ const, $\left(\Omega_{x}, \Omega_{y}, \Omega_{z}\right)$ - компоненты угловой скорости, $\Omega_{x}=\Omega_{x_{0}} \equiv 0$, при этом $y_{N}=A \sin \alpha \cos \beta_{1}+h \Omega_{z} / \nu, z_{N}=A \sin \alpha \sin \beta_{1}-h \Omega_{y} / \nu$, $s(\alpha)=B \cos \alpha, A, B, h, \sigma>0$.

\section{2. Исследуемая система с переменной диссипацией с нулевым средним.} Система (1)-(3) является динамической системой с переменной диссипацией с нулевым средним (в данном случае по углу $\alpha$ ) [2]. Это означает, что интеграл по периоду угла атаки от дивергенции ее правой части, отвечающий за изменение фазового объема (после соответствующего приведения системы), равен нулю. Система является как бы “полуконсервативной". Если $z_{1}=\Omega_{y} \cos \beta_{1}+\Omega_{z} \sin \beta_{1}, z_{2}=-\Omega_{y} \sin \beta_{1}+\Omega_{z} \cos \beta_{1}$, $\sigma h_{1} / I_{2}=H_{1}, \beta=\sigma^{2} A B / I_{2}, \sigma z_{k}=\nu w_{k}, k=1,2$ (при этом $\alpha^{\prime}=\nu \alpha \bullet / \sigma$ и т. д.), то получаем опорную систему следующего вида:

$$
\begin{aligned}
\alpha^{\prime} & =-\left(1+H_{1}\right) w_{2}+\beta \sin \alpha, \\
w_{2}^{\prime} & =\beta \sin \alpha \cos \alpha-\left(1+H_{1}\right) w_{1}^{2} \operatorname{ctg} \alpha-H_{1} w_{2} \cos \alpha, \\
w_{1}^{\prime} & =\left(1+H_{1}\right) w_{1} w_{2} \operatorname{ctg} \alpha-H_{1} w_{1} \cos \alpha, \\
\beta_{1}^{\prime} & =\left(1+H_{1}\right) w_{1} \operatorname{ctg} \alpha,
\end{aligned}
$$

При $\beta=H_{1}$ дивергенция правой части независимой подсистемы (4)-(6) ((4)-(7)) после замены переменных $w_{1}^{*}=\ln \left|w_{1}\right|$ тождественно равна нулю, что позволяет считать данную систему (системы) консервативной (консервативными).

Теорема 1. Система (4)-(7) обладает полным набором первых интегралов, являющихся элементарными трансцендентными (в смысле теории функиий комплексного переменного) функииями своих фазовых переменных. Два из них образуют полный набор первых интегралов системы (4)-(6).

Действительно, сопоставим исследуемой системе два уравнения

$$
\begin{aligned}
\frac{d w_{2}}{d \alpha} & =\frac{\beta \sin \alpha \cos \alpha-\left(1+H_{1}\right) w_{1}^{2} \frac{\cos \alpha}{\sin \alpha}-H_{1} w_{1} \cos \alpha}{-\left(1+H_{1}\right) w_{2}+\beta \sin \alpha}, \\
\frac{d w_{1}}{d \alpha} & =\frac{\left(1+H_{1}\right) w_{1} w_{2} \frac{\cos \alpha}{\sin \alpha}-H_{1} w_{1} \cos \alpha}{-\left(1+H_{1}\right) w_{2}+\beta \sin \alpha} .
\end{aligned}
$$

Работа выполнена при поддержке гранта Президента РФ для молодых докторов наук (МД-2311.2005.1) и РФФИ (гранты № 05-08-01378-a, 05-01-00401-а). 
Применяя подстановки $\tau=\sin \alpha, w_{k}=u_{k} \tau, k=1,2$, сопоставим системе (8), (9) уравнение

$$
\frac{d u_{2}}{d u_{1}}=\frac{\beta+\left(1+H_{1}\right)\left(u_{2}^{2}-u_{1}^{2}\right)-\left(H_{1}+\beta\right) u_{2}}{2\left(1+H_{1}\right) u_{1} u_{2}-\left(H_{1}+\beta\right) u_{1}}
$$

и получим цепочку инвариантных соотношений:

$$
\begin{gathered}
\frac{\left(1+H_{1}\right) u_{2}^{2}-\left(H_{1}+\beta\right) u_{2}+\left(1+H_{1}\right) u_{1}^{2}+\beta}{u_{1}}=C_{1}, \\
\frac{\left(1+H_{1}\right) w_{2}^{2}-\left(H_{1}+\beta\right) w_{2} \sin \alpha+\left(1+H_{1}\right) w_{1}^{2}+\beta \sin ^{2} \alpha}{w_{1} \sin \alpha}=C_{1}, \\
\frac{w_{2}^{2}+(1+\beta) w_{1}^{2}+\beta\left[w_{2}-\sin \alpha\right]^{2}}{w_{1} \sin \alpha}=C_{1} .
\end{gathered}
$$

Более того, легко проверить, что и числитель, и знаменатель в левой части (13) являются первыми интегралами системы (4)-(7) при $\beta=H_{1}$ :

$$
w_{2}^{2}+(1+\beta) w_{1}^{2}+\beta\left[w_{2}-\sin \alpha\right]^{2}=C_{1}^{*}, \quad w_{1} \sin \alpha=C_{2}^{*} .
$$

В случае же $\beta \neq H_{1}$ система (4)-(6) перестает быть консервативной. При этом ни числитель, ни знаменатель в левой части инвариантного соотношения (12) первыми интегралами не являются. Последний факт проверять необязательно, поскольку у системы (4)-(6) имеются притягивающие и отталкивающие предельные множества, запрещающие наличие у исследуемой системы полного набора даже непрерывных первых интегралов.

Дополнительный первый интеграл ищется из квадратуры

$$
\int \frac{d \tau}{\tau}=\int \frac{\left[\beta-\left(1+H_{1}\right) u_{2}\right] d u_{2}}{\beta-\left(H_{1}+\beta\right) u_{2}+\left(1+H_{1}\right)\left[u_{2}^{2}-U\left(u_{1}, C_{1}\right)\right]},
$$

где $U\left(u_{1}, C_{1}\right)=\left(2\left(1+H_{1}\right)\right)^{-1}\left\{C_{1} \pm \sqrt{C_{1}^{2}-4 D_{1}}\right\}$ на уровне $C_{1}>4\left(1+H_{1}\right) D_{1}$ интеграла (12), где $D_{1}=\left(1+H_{1}\right) u_{2}^{2}-\left(H_{1}+\beta\right) u_{2}+\beta$.

Общий структурный вид дополнительного первого интеграла для системы (4)-(6) таков: $\Phi_{1}\left(w_{1}, w_{2}, \sin \alpha\right)=C_{2}$.

Дополнительный первый интеграл системы (4)-(7) найдется так:

$$
\begin{gathered}
\frac{d u_{1}}{d \beta_{1}}+\left[\frac{\beta-\left(1+H_{1}\right) u_{2}}{1+H_{1}}\right]=u_{2}-\frac{H_{1}}{1+H_{1}}, \\
\sin ^{2}\left\{2\left(1+H_{1}\right)^{2}\left(\beta_{1}+C_{3}\right)\right\}=\frac{\left(2\left(1+H_{1}\right) w_{1}-2 C_{1} \sin \alpha\right)^{2}}{\left[\left(H_{1}+\beta\right)^{2}-4 \beta\left(1+H_{1}\right)+C_{1}^{2}\right] \sin ^{2} \alpha} .
\end{gathered}
$$

3. Смежная задача. Если $(\xi, \eta)$ - углы, определяющие положение пространственного маятника [3] на сфере $S^{2}$, то уравнения движения такой системы на касательном расслоении $T_{*} S^{2}$ двумерной сферы можно представить в следующем виде:

$$
\begin{gathered}
\xi^{\bullet \bullet}+\left(\beta-H_{1}\right) \xi^{\bullet} \cos \xi+\beta \sin \xi \cos \xi-\eta^{\bullet 2} \frac{\sin \xi}{\cos \xi}=0, \\
\eta^{\bullet \bullet}+\left(\beta-H_{1}\right) \eta^{\bullet} \cos \xi+\xi^{\bullet} \eta^{\bullet}\left[\frac{1+\cos ^{2} \xi}{\cos \xi \sin \xi}\right]=0 .
\end{gathered}
$$

Теорема 2. Система (4)-(6) траекторно эквивалентна системе (14), (15).

\section{Список литературы}

[1] М. В. Шамолин, Докл. РАН, 364:5 (1999), 627-629. [2] М. В. Шамолин, Докл. РАН, 371:4 (2000), 480-483. [3] М. В. Шамолин, УМН, 54:5 (1999), 181-182.

M. В. Шамолин (M. V. Shamolin)

Московский государственный университет им. М. В. Ломоносова

E-mail: shamolin@imec.msu.ru
Представлено А. В. Михалёвым Принято редколлегией 27.07.2007 